\title{
Parameter Identification on Helly's Car-Following Model
}

\author{
Roberto C. Ambrosio-Lázaro ${ }^{1}$, Luis Alberto Quezada-Téllez ${ }^{2}$, Oscar A. Rosas-Jaimes ${ }^{1}$ \\ ${ }^{1}$ Facultad de Ciencias de la Electrónica, Benemérita Universidad Autónoma de Puebla \\ Ciudad Universitaria, Puebla, México \\ roberto.ambrosio@correo.buap.mx; oscar.rosasj@correo.buap.mx \\ ${ }^{2}$ Departamento de Matemáticas Aplicadas y Sistemas, UAM-Unidad Cuajimalpa \\ Avenida Vasco de Quiroga 4871, Ciudad de México, México \\ lquezada@correo.cua.uam.mx
}

\begin{abstract}
Helly's Model is a very simple and well-known differential equation that describes the car-following phenomenon very accurately with a very intuitive approach. It involves relative distances and velocities between two cars (one in front of the other) which are the variables of the model and which are related through constants which give proportionality and consistency to it. Those constants are parameters that must be calculated in order to achieve the necessary similarity to the real behaviour that they are modelling. In this work, an identification scheme for such estimation is presented. A set of measured data taken from real driving experiments are used to calculate the values of such parameters. Then, a simulation of the velocity developed by one of the cars is performed in order to compare this simulated behaviour against the data directly measured from that same car. The results show that there the differences of both sets of data are minimal, and that the model is very well adjusted.
\end{abstract}

Keywords: Parameter Identification, Car-Following, Helly’s Model.

\section{Introduction}

The present and future increase in the global amount of cars is a very well-known fact by governments and research centres and it is evident to drivers and passengers [1,2,3,4]. This situation has had implications of social and economic order [5], legal and regulatory involvements [6], and safety issues [7]. As a complex phenomenon, it has been faced from distinct points of view of different knowledge fields. Technologically and scientifically, many mathematical models have been suggested in the last century to describe vehicles movement in a road $[8,9,10,11,12,13]$.

Velocity of the cars is a quantity directly related with the state of traffic and with the time of travel used by drivers to move from their origins to their destinations. Other involved variables are present, as density [8, 14], which is a quantity used to identify the concentration of cars in a stretch of a road. The higher this value, the higher congestion is present. And, at the same time, the space for the cars to move is smaller. The interaction among more vehicles reduce the velocity at which they move and the gaps among their physical dimensions $[9,15]$.

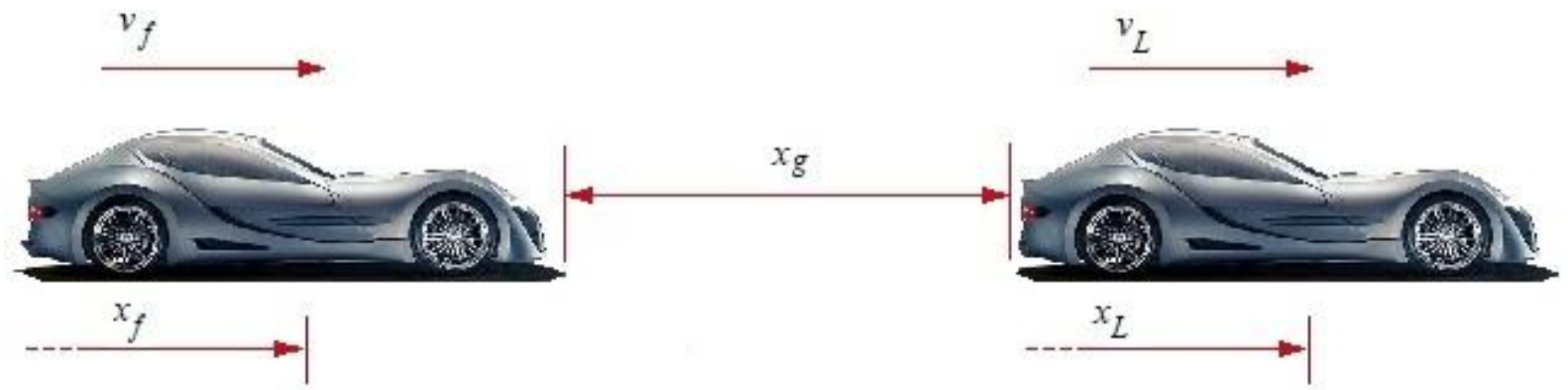

Fig. 1: Car-following variables (Felino CB7 is property of Felino Corpration).

In situations with some level of congestion, the relative velocities and distances between pairs of cars become valuable variables to model the physics of what it is happening while the traffic is flowing. In this way it is possible to identify, for two adjacent cars in a same lane, one in front (leader $L$ ) and other behind (follower $f$ ) [16, 17]. This scheme can be 
spanned to a bigger number of cars, with any one of them performing as a leader of a follower behind of it, but each one of the followers becoming a leader of another car directly located behind.

Car-following models are normally applied to those schemes with two cars as a basic unit of study [18]. One of such models is the simple Helly' model [19]. In spite of there are many others microscopic traffic models which surpass this one in accuracy, Helly's model is intuitive and very easy to use. It is pretty well formulated and it is possible to use it into the development of analyses and control designs [20]. Helly's model includes some parameters to keep the proportionality of its variables. In order to obtain a precise enough model, fitted to perform simulations of real scenarios, it is necessary to calibrate those quantities [21,22]. A practical approach to calibrate them is through a parameter identification algorithm [23] that uses variables such as relative velocities and relative distances, which can be obtained by proper devices installed in a car performing like a follower.

The main result of this document is the design of such a parameter identification algorithm, from a proper formulation of Helly's model. This model is presented in Section 2. Then, in Section 3, the identification of parameters approach is used with this model, where it is explained that sets of experimental data are needed. In Section 4 series of experiments are used to obtain those data needed to make the parameter identification algorithm work, giving detail about the way they have been carried on. The results of the identification are presented in Section 5, where a simulation of Helly's model with the value of the parameters is compared with real data, showing the accuracy of the process. At the end, some concluding remarks are pointed out for this work.

\section{Helly's Model}

The contribution of W. Helly [19] to car-following theory has been a differential equation in which the variation of the velocity of the follower car is the sum of a term involving relative velocities of such a car and that leading it, with a term that involves the relative distance between them (see Fig. 1).

$$
\frac{d v_{f}(t)}{d t}=\lambda_{v}\left[v_{L}(t)-v_{f}(t)\right]+\lambda_{x}\left[x_{L}(t)-x_{f}(t)-D(t)\right]
$$

where:

$v_{L}$ : velocity of the leader car

$v_{f}$ : velocity of the follower car

$x_{L}$ : position of the leader car

$x_{f}$ : position of the follower car

$x_{g}$ : gap between cars (bumper to bumper)

D: safety distance between cars

$\lambda_{v}$ : Sensitivity parameter for relative velocities

$\lambda_{x}$ : Sensitivity parameter for relative distances

As can be seen in Eq. (1), the acceleration of the follower vehicle is positive if the leader vehicle is traveling with a higher speed, and it is negative on the contrary, which means that the follower car is catching it up. But such an acceleration also depends on the relative position of both cars, summing some additional acceleration if the follower is far enough from the leader, but not trespassing a safety distance $\mathrm{D}$, which is observed in reality as a limit distance respected by drivers in order to be close enough to the car in front of them but keeping sufficient space to manoeuvre. In fact, drivers respond in a different manner for low velocities or high speeds. 


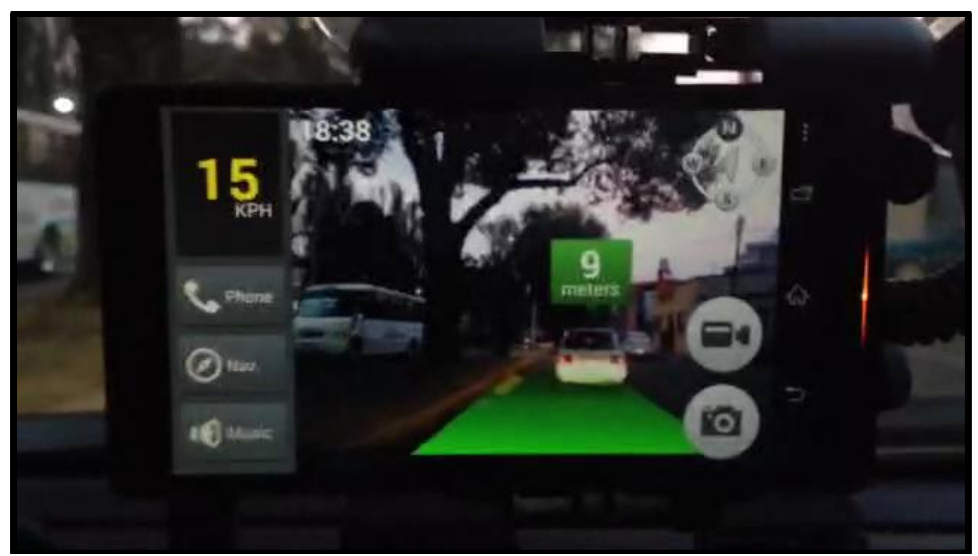

Fig. 2: Measurement of velocity of the instrumented car and of the distance to the car in front of it(IonRoad App V 1.6.3 Pro is a license of iOnRoad Limited [25]).

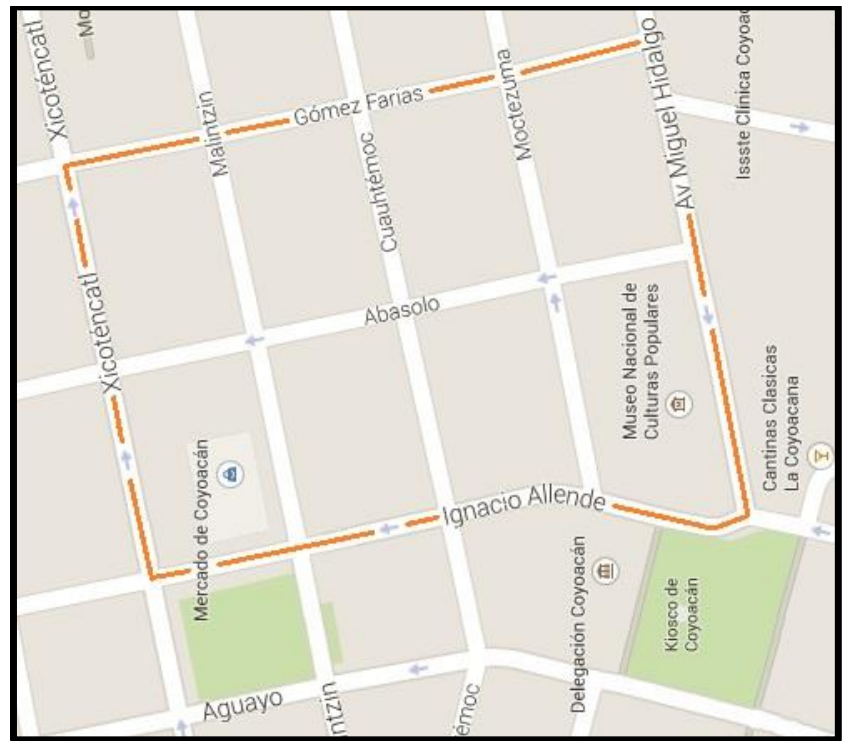

Fig. 3: Loop followed by driver in the experiment.

Those terms are kept proportional by means of two sensitivity parameters. It is assumed that both of them are quantities defined in $0<\lambda_{v}<1$ and $0<\lambda_{x}<1$ respectively. Physically they represent a measure of the follower driver's reaction to the behaviour of the leader, i.e. for those drivers with lower values of $\lambda_{v}$ and $\lambda_{x}$ correspond a less reactive behaviour, while those drivers with higher values of those parameters have a more reactive mood [10, 17, 22].

\section{Experimental Data}

Experiments with pairs of cars with dedicated devices can be conducted in order to obtain velocities, relative velocities and relative positions [17, 21, 22, 24]. There are many ways to design this type of experiments, and many of them are very expensive, because they demand material and human resources for better and more realistic results. For this case, one of the main goals is to design a set of experiments to be suitable enough to obtain satisfactory results but inexpensive enough as far as it can be possible. Only one car is used for experimentation in this case, which behaviour is that of a follower. Data to be obtained are velocity $v_{f}(t)$ and the extent of the gap $x_{g}(t)$ in front of it with respect to the car in its front while moving. An app [25] which uses the camera of a cell phone and a pattern recognition algorithm is used to measure the distance to the vehicle ahead (see Fig. 2). Unfortunately, recording of data obtained is not possible from this app, and it was necessary to record with a second camera what was viewed through the screen of the first one. 
A touristic area in Mexico City was chosen to carry on these experiments, on a day with a high attendance of people. Congestion due to heavy traffic and small gaps among cars were expected for this event. The idea was to ensure the presence of vehicles in front of the instrumented car while driving, behaving as a follower not for only one of the present cars in the road, but with many of them, supposing there is no violation to the conditions supported by Eq. (1), because this instrumented car do not surpass to any of such vehicles while driving. A loop was stablish to perform the trials and to collect data (Fig. 3).

As normally occurs, some trials were dismissed because some incidences happened: lack of familiarity of the zone and its affectation to the driver, camera positioning needing a better adjustment, sun light directly entering the lens and affecting measurements, and other similar details. At the end, four sets of data of four different trials were selected as those suitable to process.

\section{Parameter Identification Scheme}

Helly's model (1) can be rewritten as Eq. (2) shows

where:

$$
\frac{d v_{f}(t)}{d t}=\lambda_{v} v_{R}(t)+\lambda_{x} x_{R}(t)+\lambda_{d} D(t)
$$

$v_{R}=v_{L}(t)-v_{f}(t)$

$x_{R}=x_{L}(t)-x_{f}(t) \approx x_{g}$

An alternative form to Eq. (2) is Eq. (3)

$$
\frac{d v_{f}(t)}{d t}=\left[\begin{array}{lll}
v_{R}(t) & x_{R}(t) & D(t)
\end{array}\right]\left[\begin{array}{l}
\lambda_{v} \\
\lambda_{x} \\
\lambda_{d}
\end{array}\right]
$$

Eq. (3) can be easily transform in the form depicted by Eq. (4)

$$
\frac{v_{f}(t+\Delta t)-v_{f}}{\Delta t} \cong\left[\begin{array}{lll}
v_{R}(t) & x_{R}(t) \quad D(t)
\end{array}\right]\left[\begin{array}{l}
\lambda_{v} \\
\lambda_{x} \\
\lambda_{d}
\end{array}\right]
$$

A classical scheme of identification of parameters [23] tries to minimize the error $e(t)$ defined by Eq. (5)

$$
e(t)=S(t+\Delta t)-\varphi(t) \theta(t)
$$

where:

$S(t)=\left[v_{f}(t+\Delta t)-v_{f}(t)\right] / \Delta t$

$\varphi(t)=\left[\begin{array}{lll}v_{R}(t) & x_{R}(t) & D(t)\end{array}\right]$

$\theta(t)=\left[\begin{array}{lll}\lambda_{v} & \lambda_{x} & \lambda_{d}\end{array}\right]^{T}$

Eq. (5) is a calculation of how different are both sides of Eq. (4), and it is needed to look for $e(t) \rightarrow 0$ as $t \rightarrow \infty$.

Ioannou and Sun [23] describe an iterative method to ensure that $\theta(t)$ converges through

$$
\theta(t)=\theta(t-\Delta t)+\alpha P(t-\Delta t) \varphi(t)\left[1+\varphi^{T}(t) P(t-\Delta t) \varphi(t)\right]^{-1} e(t)
$$

where $\alpha$ is a gain that affects the velocity of convergence of the second term of (6), while $P(t)$ is the covariance matrix of $\varphi(t)$. Such a matrix of covariance is described by Eq. (7) 


$$
P(t)=\left[P^{-1}\left(t_{0}\right)+\varphi^{T}(t) \varphi(t)\right]^{-1}
$$

\section{Results and Discussion}

\section{1. $\lambda$ Values}

For those cases where velocity $v_{f}(t)$ or relative position $x_{R}(t)$ were missing, linear interpolation or extrapolation were used. However, from four distinct trials, only two of them were considered complete enough in duration and data collected.

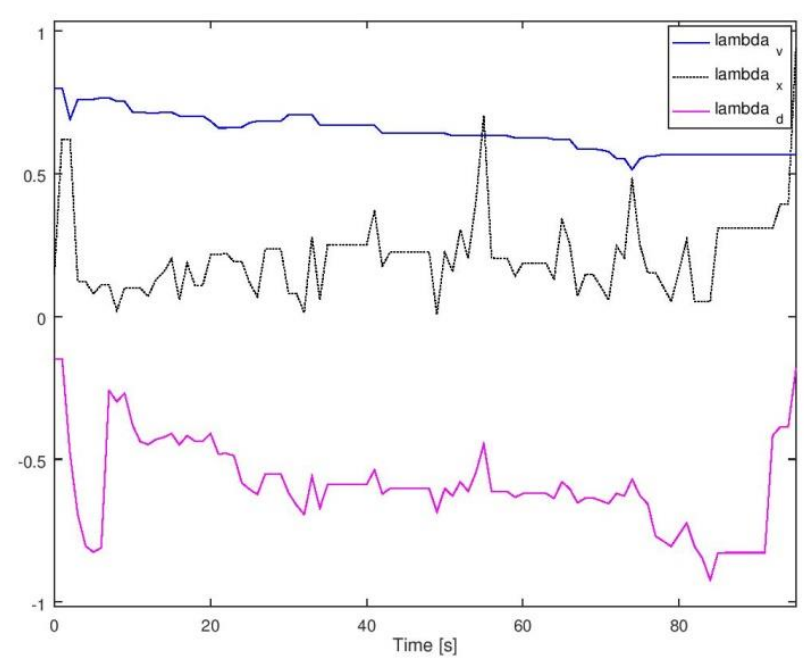

(a) Identification Evolution, trial 1

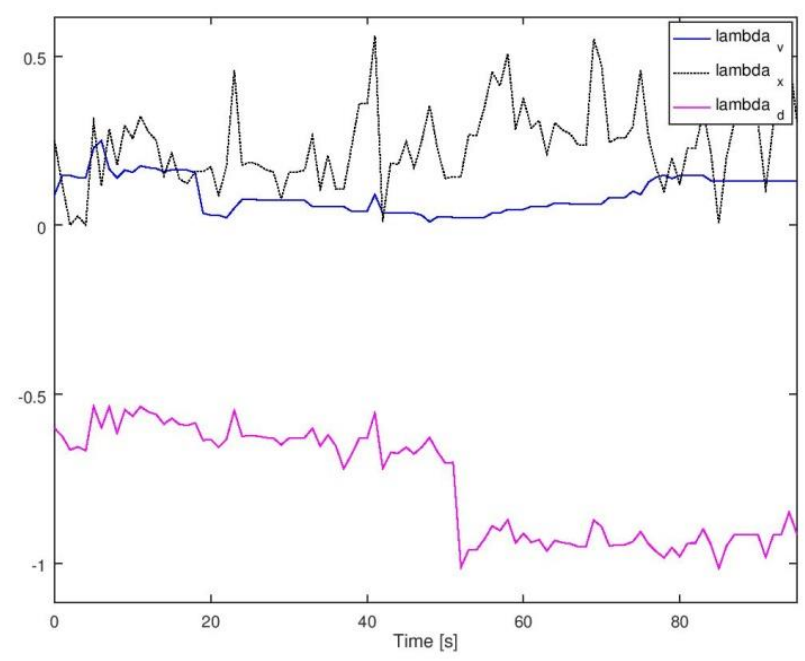

(c) Identification Evolution, trial 2

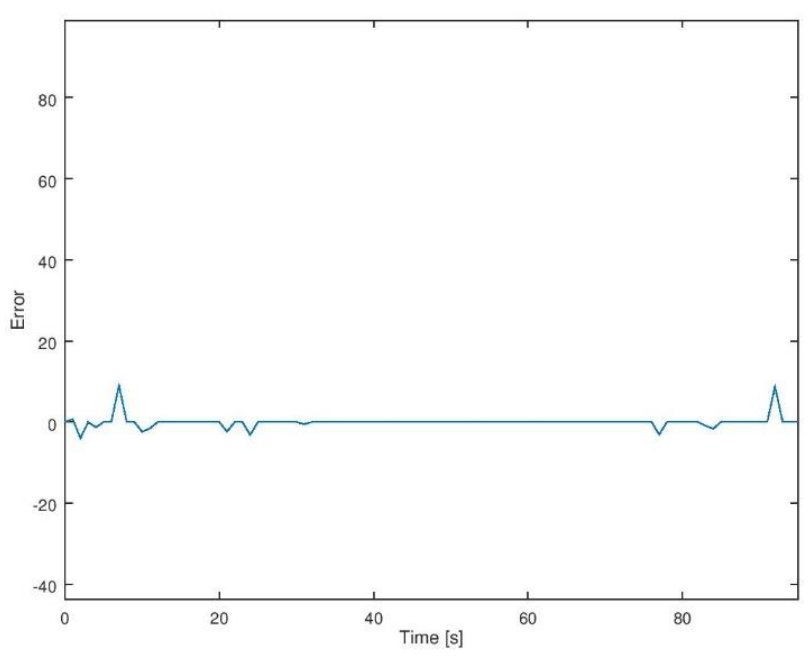

(b) Identification Error, trial 1

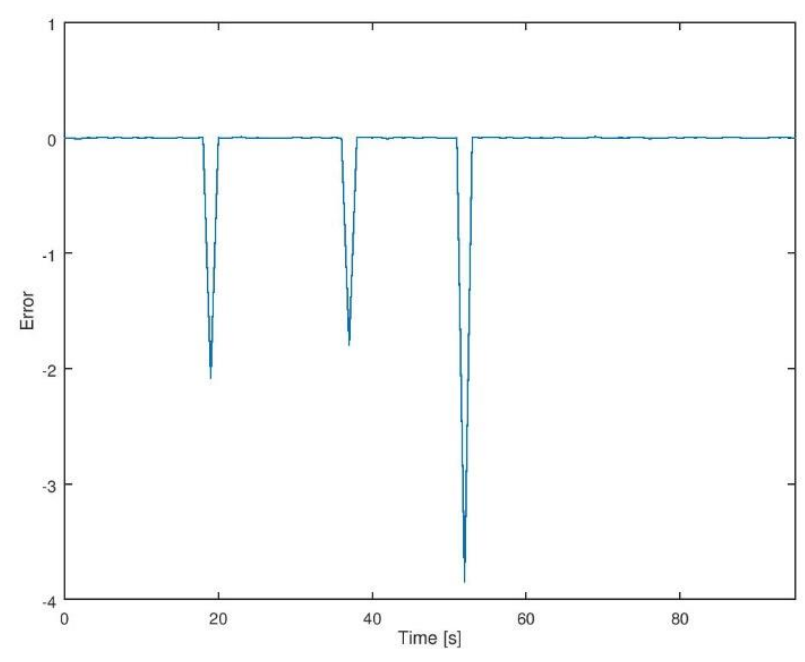

(d) Identification Error, trial 2

Fig. 4: Identification for $\lambda_{v}, \lambda_{x}$, and $\lambda_{d}$ for both experimental trials.

Identification algorithm presented in Section 3 was fed with those data sets, each one having 96 triplets $\left(t, v_{f}(t), x_{R}\right)$ for a duration of 1 minute and 36 seconds. At the same time, the value of the error $e(t)$, as described by Eq. (5), is calculated in order to watch the convergence of this scheme. Fig. 4 shows the evolution of the identification algorithm for both sets of data. Even though the parameters exhibit much variation, the error $e(t)$ of the performance, presented in Fig. $4 \mathrm{~b}$, shows a good convergence. This rather present these parameters as not entirely constants as calculations from data show. However, it is possible to see that there is a tendency to some average value in each one of these three parameters. In 
this way, it has been possible to calculate $\lambda_{v}=0.645, \lambda_{x}=0.212$ and $\lambda_{d}=-0.590$, for a first trial. For a second trial, Fig. $4 \mathrm{c}$ depicts the evolution of the algorithm when it calculates $\lambda_{v}, \lambda_{x}$ and $\lambda_{d}$. In this case convergence is also achieved, even though there are some times where it seems jeopardized, as Fig. $4 \mathrm{~d}$ shows. For this trial $\lambda_{v}=0.092, \lambda_{x}=0.236$ and $\lambda_{d}=-0.766$.

As expected, all this values are in the interval $(0,1)$. Another important detail to take into account is the sign of $\lambda_{d}$ which has been identified as negative, in close agreement with the formulation of Helly's model as described by Eq. (1) and Eq. (2), and in full consistency with the identification scheme that appears in Eq. (3).

\subsection{Simulations}

The model in Eq. (1) is now provided with those average $\lambda$ values calculated by the identification algorithm. To complete the values of the variables needed, the velocity of the leader car $v_{L}$ is calculated by Eq. (8)

with

$$
v_{L}(t)=\dot{x}_{R}(t)+v_{f}(t)
$$

$$
\dot{x}_{R}(t)=\frac{x_{R}(t+\Delta t)-x(t)}{\Delta t}
$$

With those described values, and a fix $D=3 \mathrm{~m}$, simulations are performed, trying to emulate the behaviour of the car in each of the two trials (see Fig. 5). Fig. 5a shows the velocity $v_{f m}(t)$ measured from the instrumented car (solid blue line) as well as that $v_{f s}(t)$ obtained from the calculation (dashed black line) of this same variable from Eq. (1). It can be noticed that one trajectory is very close to the other, even though the discrepancies. Fig. 5b shows a measuring of the difference between two variables through the calculation of the relative error, as defined by Eq. (10)

$$
E_{R}(t)=\frac{v_{f m}(t)-v_{f s}(t)}{v_{f s}(t)}
$$

The evolution of $E_{R}(t)$ in Fig. $5 \mathrm{~b}$ shows a very big skip little after $t=80 \mathrm{~s}$. This is caused by zero velocity values $v_{f m}=0$, because during this experimental trial the car came to stop due to traffic. This event affect the determinant of the quotient in Eq. (10), causing that big negative set of values of $E_{R}(t)$. However, once in the move again, the behaviour of this variable represents the good match of both velocities, as it can be watch in the respective figure.

For a second trail, Fig. 5c compares $v_{f m}$ and $v_{f} s$ with less accuracy. This could be expected if attention is return to the results obtained for the set of $\lambda$ values that resulted for this trial and the observations made in Subsection 5.1. However, in this case the relative error $E_{R}(t)$ between these two data sets is more steady as Fig. 5d shows, and because fortunately the instrumented car never came to stop for this trial, as it was the case for the first event.

The way in which data were collected includes, as mentioned early, important amounts of disturbances and noise and a lack of more accuracy. However, it can be noticed that the identification, as well as the simulations, are behaving very well in spite of such flaws, which can be surpassed if better devices are used. We stress, on the other hand, that the identification algorithm is quite stable and useful to perform this calibration.

\section{Conclusion}

To be operative, those parameters that appear on Hellys model must be calibrated. In this work, a one-car experiment has been developed to obtain those necessary data sets to perform the identification needed. This experiment needed the minimal costs in material and human resources. The identification has obtained $\lambda$ values for Helly's model which later have been used in a simulation, together with the other data of variables like the velocity of the car used by the experiment, and the relative distance to the other vehicles that subsequently were in front of the instrumented car. That simulation resulted in a similar trajectory of the simulated velocity with respect to the measured 
velocity. Even though this results are quite interesting from the point of view of calibration, they lack of precision which is possible to improve by using more accurate measuring devices.

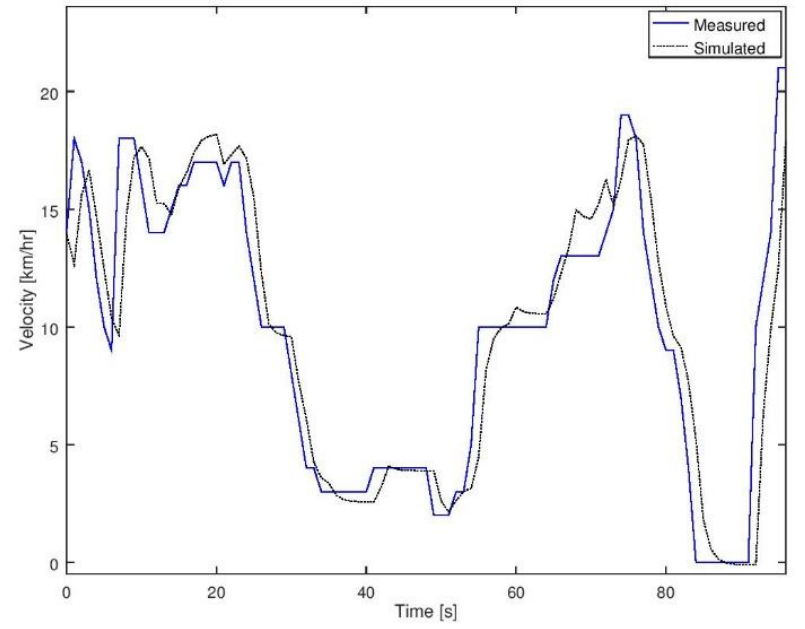

(a) Comparison between measured and simulated $v_{f}$, trial 1

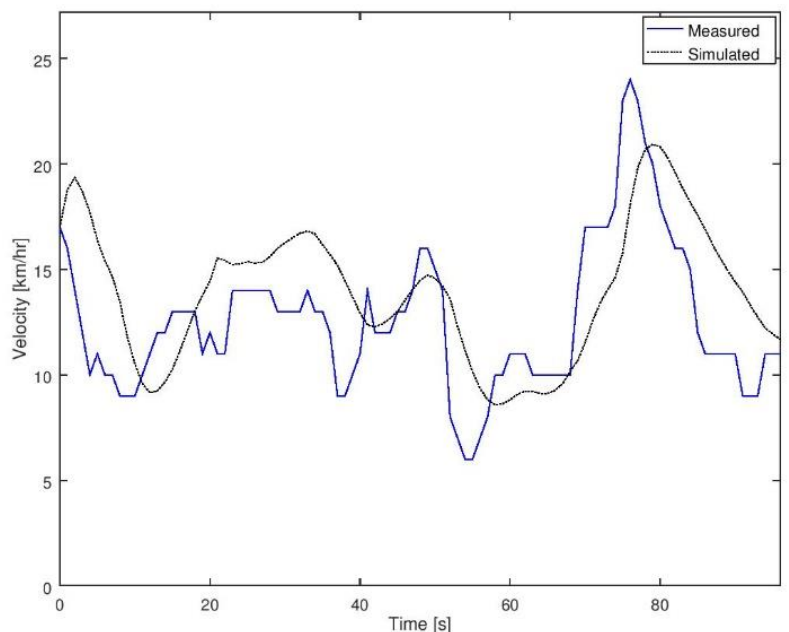

(c) Comparison between measured and simulated $v_{f}$, trial 1

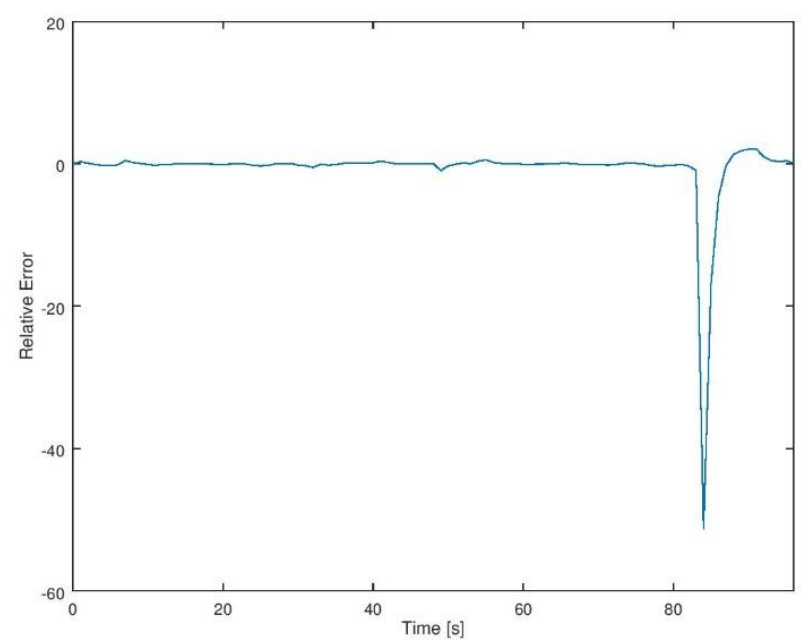

(b) Relative error between measured and simulated $v_{f}$, trial 1

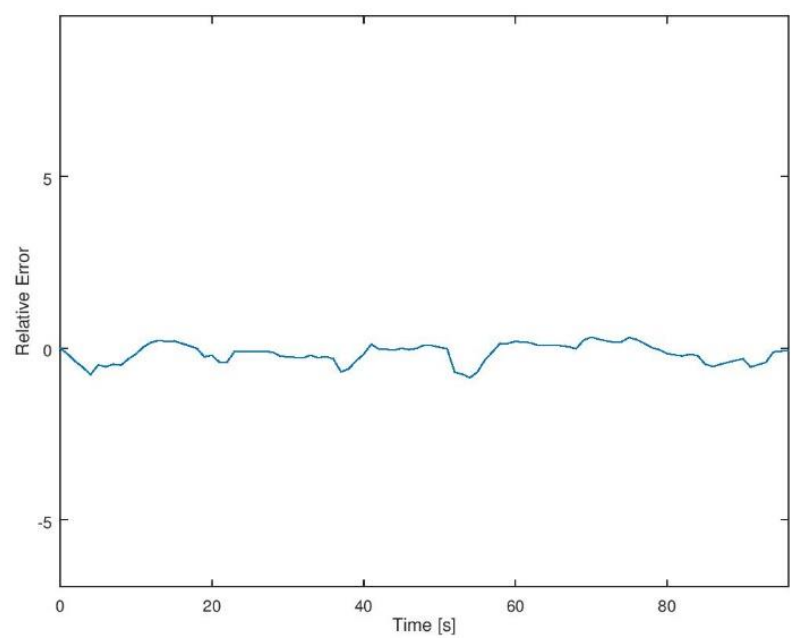

(d) Relative error between measured and simulated $v_{f}$, trial 2 Fig. 5: Simulation performed with the sets of data obtained from the experiments and the parameter identification.

\section{Acknowledgements}

This work has been supported by SEP-PRODEP project, No. 511-6/17-8017.

\section{References}

[1] Natural Resources Canada (2017, November 17). 2009 Canadian Vehicle Survey. Summary Report. [Online]. Available: http://oee.nrcan.gc.ca/publications/statistics/cvs/2009/index.cfm

[2] Transportation Research Board (2017, October 26), 2010 Highway Capacity Manual. [Online]. Available: http://hcm.trb.org/?qr=1

[3] Texas A\&M Transportation Institute (2017, November 17), 2015 Urban Mobility Scorecard. [Online]. Available: http://mobility.tamu.edu/ums

[4] Transportation Research Board (2017, November 15), 2016 Highway Capacity Manual [Online]. Available: http://hcm.trb.org/?qr=1http://trrjournalonline.trb.org/doi/book/10.5555/9780309441483 
[5] A. Downs, "Stuck in Traffic: Coping with Peak-Hour Traffic Congestion," The Brookings Institution: Washington, DC., 1992.

[6] V. Islas-Rivera, "Implementing Sustainable Urban Travel Policies in Mexico," International Transport Forum Discussion Papers, no. 2011/14, OECD Publishing, Paris, 2011.

[7] S. Miaou and J. Jin, "Bayessian ranking of sites for engineering safety improvements: Decision parameter, treatability concept, statistical criterion, and spatial dependence," Accident Analysis and Prevention, vol. 37, pp. 699-720, 2005.

[8] C. F. Daganzo, Fundamentals of Transportation and Traffic Operations. Pergamon, 1997.

[9] M. Schonhof and D. Helbing, "Empirical Features of Congested Traffic States and Their Implications for Traffic Modelling," Transportation Science, vol. 41, no. 2, pp. 135-166, 2007.

[10] P. A. Ioannou, Y. Wang, and H. Chang, "Chapter 13. Modeling, Simulation and Control of Transportation Systems," in Modeling and Control of Complex Systems, CRC Press, pp. 407-437, 2008.

[11] J. Ortúzar and L. G. Willumsen, Modelling Transport, 4th ed., John Wiley \& Sons, 2011.

[12] M. J. Beckmann, "Traffic congestion and what to do about it," Transportmetrica B: Transport Dynamics, no. 1, pp. 103-109, 2013.

[13] F. Van Wageningen-Kessels, H. van Lint, K. Vuik and S. Hoogendoorn, "Genealogy of traffic flow models," EURO Journal on Transportation and Logistics, vol. 4, no. 4, 445-473, 2015.

[14] V. A. Thamizh and G. Dhivya, "Measuring heterogeneous traffic density," International Journal of Engineering and Applied Sciences, vol. 6, no. 3, pp 144-148, 2010.

[15] M. Treiber, A. Hennecke and D. Helbing, "Congested traffic states in empirical observations and microscopic simulations," Physical Review E, vol. 62, no. 2, pp. 1805-1824, 2000.

[16] A. Kesting and M. Treiber, "Calibrating Car-Following Models by Using Trajectory Data: Methodological Study," Transportation Research Record, 2008. https://arxiv.org/abs/0803.4063v1

[17] O. Rosas-Jaimes, O. Luckie-Aguirre and J. C. López-Rivera, "Identification and Analysis of a Sensibility Parameter of a Microscopic Traffic Model," in Road Safety and Simulation Conference RSS 2013, Rome, Italy, 2013.

[18] S. Panwai and H. Dia, "Comparative Evaluation of Microscopic Car-Following Behavior," IEEE Transactions on Intelligent Transportation Systems, vol. 6, no. 3, pp. 314-325, 2005.

[19] W. Helly, "Simulation of Bottlenecks in Single Lane Traffic Flow," in Proceedings of the Symposium on Theory of Traffic Flow, Research Laboratories, General Motors, Elsevier, 1959.

[20] O. Rosas-Jaimes, L. Quezada-Téllez and G. Fernández-Anaya, "Control Designs and Stability Analyses for Carfollowing Helly's Model," Transportmetrica B: Transport Dynamics, (submitted).

[21] S. B. Chung, K. H. Song, S. Y. Hong and S. Y. Kho, "Development of sensitivity term in car-following model considering practical driving behaviour of preventing rear end collision," Journal of the Eastern Asia Society for Transportation Studies, no. 6, pp. 1354-1367, 2005.

[22] O. Rosas-Jaimes, O. Luckie-Aguirre, O. and J. C. López-Rivera, "Calibration and Comparison of Two Microscopic Traffic Models," in Proceedings of XVI Congreso Latinoamericano de Control Automático, CLCA 2014, pp. 10061011, Cancún, Quintana Roo, México, 2014.

[23] P. A. Ioannou and J. Sun, Robust Adaptive Control. Prentice Hall, Englewood Cliffs, NJ, 1996.

[24] E. Brockfeld and P. Wagner, "Calibration and Validation of Microscopic Traffic Flow Models," in: S.P. Hoogendoorn, S. Luding, P. H. L. Bovy, M. Schreckenberg, D. E. Wolf (eds) Traffic and Granular Flow '03. Springer, Berlin, Heidelberg, 2005.

[25] Mobile application software, iOnRoad Smart Phone Smarter Driver (Version 1.6.3p) [Online]. Available: http://www.ionroad.com, 2017. 